\title{
A Fuzzy Technique for Solving Rough Interval Multiobjective Transportation Problem
}

\author{
Mohamed S. Osman \\ Prof. of Pure mathematics \\ and Operations \\ Researches, El-Asher \\ University \\ Egypt
}

\author{
Mahmoud M. El- \\ Sherbiny \\ Prof. of Operations \\ Researches Institute of \\ Statistical Studies \& \\ Researches, \\ Cairo University Egypt
}

\author{
Hamdeen A. Khalifa \\ Dr. of Operations \\ Researches \\ Institute of Statistical \\ Studies \& Researches, \\ Cairo University Egypt
}

\author{
Hanan H. Farag \\ Researcher in Institute of \\ Statistical Studies\& \\ Researches, \\ Cairo University, Egypt
}

\begin{abstract}
In this paper the rough interval multiobjective transportation problem (RIMOTP) is presented and its solution procedure is introduced. The concept of solving the interval multiobjective transportation problem is applied for solving RIMOTP. So, The rough interval in the objective function and the constrains, is represented by three different models and such models are solved by using fuzzy programming technique based on the right limit, the center and the half-width of each rough interval using possibly region. Numerical examples are provided to illustrate the solution procedure of three possible types of the original problem.
\end{abstract}

\section{General Terms}

Fuzzy Programming Technique, Transportation Problem, rough interval multiobjective transportation

\section{Keywords}

Rough interval, multiobjective programming, transportation problem, fuzzy programming technique, Pareto optimal solution

\section{INTRODUCTION}

The theory of rough sets is presented by Pawlak [12]. The rough programming is discussed by many authors $[5,9$, and 15]. Abd El-Wahed [1, 7] presented a fuzzy programming approach to determine the optimal compromise solution of a multiobjective transportation problem. A fuzzy technique is used to solve the multiobjective transportation problems with interval cost [4]. Ammar et al [3] proposed a method to solve rough interval multiobjective transportation problem based on weighting method and separation method. Mohanty and Dash [8] presented the uncertainty distribution to solve rough transportation problem. El-Sisy [5] presented the duality of multiobjective rough convex programming problem under uncertainty. $\mathrm{Xu}$ and Tao [14] introduced a class of rough multiobjective programming problem. Bit et al [2] developed a procedure applying fuzzy programming technique for solving the multi-criteria decision making transportation problem.

In this paper, the concept of solving the conventional interval linear programming problem combined with the fuzzy technique is used to solve the rough interval multiobjective transportation problem. Such technique is used to deal with three different types of rough interval multiobjective transportation problem. The remaining of the paper unfolds as follows: In Section 2, rough interval definition is presented. In section 3, Multiobjective Transportation Problem is illustrated. Section 4, The Proposed Approach is devoted to numerical examples for the illustration. .

\section{ROUGH INTERVAL}

Definition 1: Let $w$ denote a closed and bounded set of numbers. A rough interval $W^{R}=\left[w^{L}: w^{U}\right]$ is defined as an interval with known lower $w^{L}=\left[w^{a}, w^{b}\right]$ and upper bound $w^{U}=\left[w^{c}, w^{d}\right]$ but unknown distribution information for $w$. and $w^{L} \subseteq w^{U}$. When $w^{L}=w^{U} ; W^{R}$ becomes a conventional interval, i.e. $W^{R}=w^{L}=w^{U}$ [13].

Definition 2: Let $* \in\{+,-, \times, \div\}$ be a binary operation on rough interval $W^{R}$ and $Z^{R}$ when $W^{R}, Z^{R} \geq 0$ we have $w^{L}, w^{U}, Z^{L}$ and $\mathrm{ZU}$ are conventional intervals, the above operations can be further transferred to the following functions if letting $w^{L}=\left[w^{a}, w^{b}\right], w^{U}=\left[w^{c}, w^{d}\right], Z^{L}=\left[Z^{a}\right.$, $\left.Z^{b}\right], Z^{U}=\left[Z^{c}, Z^{d}\right]$ where $w^{a}, w^{b}, w^{c}, w^{d}, Z^{a}, Z^{b}, Z^{c}$ and $Z \mathrm{~d}$ are deterministic numbers denoting the lower and upper bounds of $w^{L}, w^{U}, Z^{L}$ and $Z^{U}$

$$
\begin{aligned}
& W^{R}+Z^{R}=\left(\left[w^{a}+Z^{a}, w^{b}+Z^{b}\right]:\left[w^{c}+Z^{c}, w^{d}+Z^{d}\right]\right) \\
& W^{R}-Z^{R}=\left(\left[w^{a}-Z^{a}, w^{b}-Z^{b}\right]:\left[w^{c}-Z^{c}, w^{d}-Z^{d}\right]\right) \\
& W^{R} \times Z^{R}=\left(\left[w^{a} \times Z^{a}, w^{b} \times Z^{b}\right]:\left[w^{c} \times Z^{c}, w^{d} \times Z^{d}\right]\right) \\
& W^{R} \div Z^{R}=\left(\left[w^{a} \div Z^{a}, w^{b} \div Z^{b}\right]:\left[w^{c} \div Z^{c}, w^{d} \div Z^{d}\right]\right) \\
& \text { where } Z^{a}, Z^{b}, Z^{c} \text { and } Z^{d} \neq 0 \text { in }\{\div\} \text { binary operation[6]. }
\end{aligned}
$$

\section{MULTIOBJECTIVE}

\section{TRANSPORTATION PROBLEM}

Multiobjective Transportation Problem (MOTP) can be classified into three different types based on the certainty of its coefficient. The traditional MOTP where all the coefficients in the objective function and the entire constrains are deterministic values. The second type is the interval multiobjective transportation problem (IMOTP) where the coefficients of the model represented by interval values. The third type is rough interval multiobjective transportation problem (RIMOTP) where the coefficients of the model are represented by rough interval values. The following section will illustrate the mathematical model of each type.

\subsection{The Deterministic Multiobjective Transportation Problem}

The Deterministic multiobjective transportation problem can be defined as: Suppose that [8] there are $m$ sources and $n$ 
destinations. Let $a_{i}$ be the number of supply units available at sources $i(i=1,2, \ldots, m)$ and let $b j$ the number of demands units required at destination $\mathrm{j}(j=1,2, \ldots, n)$. Let cij represents the unit transportation cost for transportation the units from source $i$ to destination $j$. The target is to determine the number of units to be transported from source $i$ to destination $\mathrm{j}$, so that the total transportation cost is minimum. Let $x_{i j}$ be the decision variable which denotes the number of units shipped from sources $i$ to destination $j$.

$\operatorname{Min} f^{k}(x)=\sum_{i=1}^{m} \sum_{j=1}^{n} c_{L i j}^{k} x_{i j}$ where $k=1,2, \ldots, K$

subject to

$$
\begin{aligned}
& \sum_{j=1}^{n} x_{i j}=a_{i}, i=1,2, \ldots, m \\
& \sum_{i=1}^{m} x_{i j}=b_{j}, j=1,2, \ldots, n \\
& \sum_{i=1}^{m} a_{i}=\sum_{j=1}^{n} b_{j}, i=1,2, \ldots, m, j=1,2, \ldots, n \\
& x_{i j} \geq 0
\end{aligned}
$$

The weighting method [2], $\varepsilon$-constrained [5], and fuzzy technique [16] can be used to solve model (1).

\subsection{Interval Multiobjective Transportation \\ Problem}

The formulation of interval multiobjective transportation (IMOTP) problem of minimizing interval cost of $K^{\text {th }}$ objectives function under constrains of interval sources $\left[a_{i}^{L}: a_{i}^{U}\right]$ and interval demands $\left[b_{j}^{L}: b_{j}^{U}\right]$ where $L, U$ are the lower and upper of each conventional interval [4]:

$\operatorname{Min}^{k}(x)=\sum_{i=1}^{m} \sum_{j=1}^{n}\left[c_{i j}^{k L}, c_{i j}^{k U}\right] x_{i j}$ where $k=1,2, \ldots, K$

subject to

$$
\begin{aligned}
& \sum_{j=1}^{n} x_{i j}=\left[a_{i}^{L}, a_{i}^{U}\right], i=1,2, \ldots, m \\
& \sum_{i=1}^{m} x_{i j}=\left[b_{j}^{L}, b_{j}^{U}\right], j=1,2, \ldots, n \\
& \sum_{i=1}^{m}\left[a_{i}^{L}, a_{i}^{U}\right]=\sum_{j=1}^{n}\left[b_{j}^{L}, b_{j}^{U}\right], i=1,2, \ldots, m, j=1,2, \ldots, n \\
& x_{i j} \geq 0
\end{aligned}
$$

Where $\left[c_{i j}^{k L}: c_{i j}^{k U}\right]$ : the lower and upper cost of good which to be transported from the source $i$ to destination $j$ in objective function $k$.

There are special types of IMOTP can be derived from its general form. In the first type, the objective functions' parameters are denoted by conventional intervals while the remaining parameters of the model (the supplies capacities and destination demands) are deterministic. This type can be represented as in (3)

$$
\left.\begin{array}{c}
\operatorname{Min}^{k}(x)=\sum_{i=1}^{m} \sum_{j=1}^{n}\left[c_{L i j}^{k}, c_{U i j}^{k}\right] x_{i j} \text { where } k=1,2, \ldots, K \\
\text { subject to } \\
\sum_{j=1}^{n} x_{i j}=a_{i}, i=1,2, \ldots, m \\
\sum_{i=1}^{m} x_{i j}=b_{j}, j=1,2, \ldots, n \\
\sum_{i=1}^{m} a_{i}=\sum_{j=1}^{n} b_{j}, i=1,2, \ldots, m, j=1,2, \ldots, n \\
x_{i j} \geq 0 \forall i, j, i=1,2, \ldots, m, j=1,2, \ldots, n
\end{array}\right\}
$$

In the second type, the objective functions' parameters are deterministic while the supplies capacities and destination demands are denoted by conventional intervals. The linear model of such problem can be presented as in (4)

$$
\begin{aligned}
& \text { Min } f^{k}(x)=\sum_{i=1}^{m} \sum_{j=1}^{n} c_{i j}^{k} x_{i j} \text { where } k=1,2, \ldots, K \\
& \text { subject to } \\
& \sum_{j=1}^{n} x_{i j}=\left[a_{i}^{L}, a_{i}^{R}\right], i=1,2, \ldots, m \\
& \sum_{i=1}^{m} x_{i j}=\left[b_{j}^{L}, b_{j}^{R}\right], j=1,2, \ldots, n \\
& \sum_{i=1}^{m}\left[a_{i}^{L}, a_{i}^{R}\right]=\sum_{j=1}^{n}\left[b_{j}^{L}, b_{j}^{R}\right], i=1,2, \ldots, m, j=1,2, \ldots, n \\
& x_{i j} \geq 0
\end{aligned}
$$

In order to solve the IMOTP, the separation method [3] is used. The original model can be represented by two different models which are the lower bound approximation model and the upper bound approximation model and solve each model separately. After that the solutions of both models are collected together to represent the interval solution for the problem. While the deterministic solution is targeted the fuzzy technique is applied to deal with the problem [11].

\subsection{Rough Interval Multiobjective \\ Transportation Problem}

The general rough interval multiobjective transportation problem (RIMOTP) can be stated by (5) as follows:

$$
\begin{gathered}
\operatorname{Min} f^{k}(x)=\sum_{i=1}^{m} \sum_{j=1}^{n}\left[\left[c_{i j}^{a k}, c_{i j}^{b k}\right]:\left[c_{i j}^{c k}, c_{i j}^{d k}\right]\right] x_{i j} \quad \text { where } k=1,2, \ldots K \\
\text { subject to } \\
\sum_{j=1}^{n} x_{i j}=\left[\left[a_{i}^{a}, a_{i}^{b}\right]:\left[a_{i}^{c}, a_{i}^{d}\right]\right] \quad i=1,2, \ldots m \\
\sum_{i=1}^{m} x_{i j}=\left[\left[b_{j}^{a}, b_{j}^{b}\right]:\left[b_{j}^{c}, b_{j}^{d}\right]\right] \quad j=1,2, \ldots n \\
\sum_{j=1}^{n}\left[\left[a_{i}^{a}, a_{i}^{b}\right]:\left[a_{i}^{c}, a_{i}^{d}\right]\right]=\sum_{i=1}^{m}\left[\left[b_{j}^{a}, b_{j}^{b}\right]:\left[b_{j}^{c}, b_{j}^{d}\right]\right] \\
x_{i j} \geq 0
\end{gathered}
$$

Where the supply, demand, and objective functions are donated by rough intervals $a_{i}^{R I}=\left[\left[\mathrm{a}_{i}^{a}, \mathrm{a}_{i}^{b}\right]:\left[\mathrm{a}_{i}^{c}, \mathrm{a}_{i}^{d}\right]\right]$, $b_{j}^{R I}=\left[\left[b_{j}^{a}, b_{j}^{b}\right]:\left[b_{j}^{c}, b_{j}^{d}\right]\right]$ and, $f^{R I(k)}=$ 
$\left[\left[f^{a k}, f^{b k}\right]:\left[f^{c k}, f^{d k}\right]\right]$ respectively and

$1,2, \ldots, K$ is the number of objectives.

The rough intervals

$\left[\left[c_{i j}^{a k}, c b_{i j}^{b k}\right]:\left[c_{i j}^{c k}, c_{i j}^{d k}\right]\right] k=1,2, \ldots, K$ are denoted the uncertain costs for the transportation problem. The source parameter lies between lower approximations interval LAI $\left[a^{a}, a_{i}^{b}\right]$ (surly) and upper approximation interval UAI $\left[a_{i}^{c}, a_{i}^{d}\right]$ (possibly). Similarly, destination parameter lies between lower approximation LAI $\left[b_{j}^{a}, b_{j}^{b}\right]$ (surly) and upper approximation $\quad U A I\left[b_{j}^{c}, b_{j}^{d}\right]$ (possibly)

Two special types of (RIMOTP) can be derived from the general form. In the first type

$\operatorname{Min} f^{k}=\sum_{i=1}^{m} \sum_{j=1}^{n}\left[\left[c_{i j}^{a k}, c_{i j}^{b k}\right]:\left[c_{i j}^{c k}, c_{i j}^{d k}\right]\right] x_{i j} \quad$ where $k=1,2, \ldots K$

subject to

$$
\begin{aligned}
\sum_{j=1}^{n} x_{i j}=a_{i} \quad, i=1,2, \ldots, m \\
\sum_{i=1}^{m} x_{i j}=b_{j}, j=1,2, \ldots, n \\
\sum_{i=1}^{m} a_{i}=\sum_{j=1}^{n} b_{j} \\
x_{i j} \geq 0
\end{aligned}
$$

The objective functions' parameters are denoted by rough interval while the remaining parameters of the model (the supplies capacities and destination demands) are deterministic values as in (6).

In the second type, the objective functions' parameters are deterministic while the supplies capacities and destination demands are denoted by rough intervals as in (7).

$\operatorname{Min}^{k}(x)=\sum_{i=1}^{m} \sum_{j=1}^{n} c_{i j}^{k} x_{i j} \quad$ where $k=1,2, \ldots K$

subject to

$$
\begin{aligned}
& \sum_{j=1}^{n} x_{i j}=\left[\left[a_{i}^{a}, a_{i}^{b}\right]:\left[a_{i}^{c}, a_{i}^{d}\right]\right] \quad i=1,2, \ldots, m \\
& \sum_{i=1}^{m} x_{i j}=\left[\left[b_{j}^{a}, b_{j}^{b}\right]:\left[b_{j}^{c}, b_{j}^{d}\right]\right] j=1,2, \ldots, n \\
& \sum_{j=1}^{n}\left[\left[a_{i}^{a}, a_{i}^{b}\right]:\left[a_{i}^{c}, a_{i}^{d}\right]\right]=\sum_{i=1}^{m}\left[\left[b_{j}^{a}, b_{j}^{b}\right]:\left[b_{j}^{c}, b_{j}^{d}\right]\right] \\
& x_{i j} \geq 0
\end{aligned}
$$

Definition 3 (Pareto surely-feasible solution) A solution is defined as surely-feasible solution if $\mathrm{x}$ belongs to the lower approximation of the feasible set.

Definition 4 (Pareto possibly-feasible solution): A solution is defined as possibly-feasible solution if $\mathrm{x}$ belongs to the upper approximation of the feasible set

\section{THE PROPRSED APPROACH}

The proposed approach is built based on the concept of:

Step 1: Convert the rough objective into deterministic objective as in section 4.1 .
Step 2: Convert the rough constraints into deterministic constraints as in section 4.2.

Step 3: Construct the payoff Table by solving each objective function individually under the set of constrains and calculate the values of the other objectives at the resulted solution.

Step 4: Define the maximum and the minimum value of each objective function from the payoff Table.

Step 5: Construct the membership function of each objective function using (11),(12), (13) and (14).

Step 6: Construct the linear model defined by (15).

Step 7: Solve the linear model resulted from Step 6

\subsection{Converting Rough Interval Objective to Deterministic Objective}

In order to solve the conventional interval multiobjectve programming many Authors converted it to 2 separate models and solve it separately and collecting the solution of the two in on solution [11]. Based on such concept, the rough interval multiobjective problem can be converted into a linear multiobjectve programming as in (8).

$$
\begin{aligned}
& \text { Min } \quad f_{\text {ULRI }}^{k}(x)=\sum_{i=1}^{m} \sum_{j=1}^{n} c_{i j}^{k b} x_{i j} \\
& \text { Min } \quad f_{\text {CNLRI }}^{k}(x)=\sum_{i=1}^{m} \sum_{j=1}^{n} c_{i j}^{k C N L} x_{i j} \\
& \text { Min } \quad f_{\text {UURI }}^{k}(x)=\sum_{i=1}^{m} \sum_{j=1}^{n} c_{i j}^{k d} x_{i j} \\
& \text { Min } \quad f_{\text {CNURI }}^{k}(x)=\sum_{i=1}^{m} \sum_{j=1}^{n} c_{i j}^{k C N U} x_{i j}
\end{aligned}
$$

where $k=1,2, \ldots, K$

$$
\begin{aligned}
& f_{U L R I}^{k}=\sum_{i=1}^{m} \sum_{j=1}^{n}\left(w L^{k}+c L^{k c n L}\right) \mathrm{X}_{i j}=\sum_{i=1}^{m} \sum_{j=1}^{n} c_{i j}^{b} x_{i j}, \\
& f_{C N L R I}^{k}=\sum_{i=1}^{m} \sum_{j=1}^{n}((a+b) \div 2) X_{i j}, \\
& \mathrm{~W} L^{k}=(\mathrm{b}-\mathrm{a}) \div 2 \quad \text { and } C^{k C N L}=(\mathrm{b}+\mathrm{a}) \div 2 \\
& f_{U U R I}^{k}=\sum_{i=1}^{m} \sum_{j=1}^{n}\left(w U^{k}+c U^{k C N U}\right) \mathrm{X}_{i j}=\sum_{i=1}^{m} \sum_{j=1}^{n} c_{i j}^{d} x_{i j}, \\
& f_{C N U R I}^{k}=\sum_{i=1}^{m} \sum_{j=1}^{n}((c+d) \div 2) X_{i j}, \\
& \mathrm{~W} U^{k}=(\mathrm{d}-c) \div 2 \text { and } C^{k C N U}=(\mathrm{d}+c) \div 2
\end{aligned}
$$

Where:

$f^{K}{ }_{U L R I}$ : the objective of the upper for lower approximation rough interval

$f_{C N L R I}^{K}$ : the objective of the center for lower approximation rough interval

$f^{K}{ }_{U U R I}$ : the objective of the upper for upper approximation rough interval

$f_{C N U R I}^{K}$ the objective of the center for upper approximation rough interval 


\subsection{Converting Rough Interval Constraint \\ to Deterministic Constraint}

Consider the following multiobjective transportation problem as illustrated in (9.a-9.f):

$\operatorname{Min} f^{R I(k)}(x)=\sum_{i=1}^{m} \sum_{j=1}^{n} c_{i j}^{R l(K)} x_{i j}$, where $R I=a, b, c, d, k=1,2, \ldots, K$

subject to

$$
\begin{aligned}
& \sum_{j=1}^{n} x_{i j} \leq a_{i}^{b}, \sum_{j=1}^{n} x_{i j} \geq a_{i}^{a}, i=1,2, \ldots, m \\
& \sum_{j=1}^{n} x_{i j} \leq a_{i}^{d}, \sum_{j=1}^{n} x_{i j} \geq a_{i}^{c}, i=1,2, \ldots, m \\
& \sum_{i=1}^{m} x_{i j} \leq b_{j}^{b}, \sum_{i=1}^{m} x_{i j} \geq b_{j}^{a}, j=1,2, \ldots, n \\
& \sum_{i=1}^{m} x_{i j} \leq b_{j}^{d}, \sum_{i=1}^{m} x_{i j} \geq b_{j}^{c}, j=1,2, \ldots, n \\
& \sum_{i=1}^{m} a_{i}^{a}=\sum_{j=1}^{n} b_{j}^{a}, \sum_{i=1}^{m} a_{i}^{b}=\sum_{j=1}^{n} b_{j}^{b}, \sum_{i=1}^{m} a_{i}^{c}=\sum_{j=1}^{n} b_{j}^{c}, \sum_{i=1}^{m} a_{i}^{d}=\sum_{j=1}^{n} b_{j}^{d}
\end{aligned}
$$

$$
x_{i j} \geq 0 \text {. }
$$

By studying model (9) we can find that constraints (9.b) are dominated by constraints (9.c). Also, constraints (9.d) are dominated by constraints (9.e). So, the model (9) can be rewritten as in (10).i.e possibly region.

$$
\begin{gathered}
\operatorname{Min} f^{R I(k)}=\sum_{i=1}^{m} \sum_{j=1}^{n} c_{i j}^{R I(K)} x_{i j}, \text { where } R I=a, b, c, d, k=1,2, \ldots, K \\
\text { subject to } \\
\sum_{j=1}^{n} x_{i j} \leq a_{i}^{d}, \sum_{j=1}^{n} x_{i j} \geq a_{i}^{c}, i=1,2, \ldots, m \\
\sum_{j=1}^{n} x_{i j} \leq b_{j}^{d}, \sum_{j=1}^{n} x_{i j} \geq b_{j}^{c}, j=1,2, \ldots, n \\
\sum_{i=1}^{m} a_{i}^{c}=\sum_{j=1}^{n} b_{j}^{c}, i=1,2, \ldots, m, j=1,2, \ldots, n \\
\sum_{i=1}^{m} a_{i}^{d}=\sum_{j=1}^{n} b_{j}^{d}, i=1,2, \ldots, m, j=1,2, \ldots, n \\
x_{i j} \geq 0 \forall i, j .
\end{gathered}
$$

\subsection{Membership Function}

Assuming that membership functions are linear, the linear membership for minimization of $f_{U L R I}^{K}, f_{C N L R I}^{K} f_{U U R I}^{K}$, and $f_{C N U R I}^{K}$ are given by (11), (12), (13), and (14) respectively [16].

$$
\mu_{k}\left(f_{U L R I}^{k}\right)=\left\{\begin{array}{lll}
1 & \text { if } & f_{\text {ULRI }}^{k} \leq L f_{\text {ULRI }}^{k} \\
1-\frac{f_{\text {ULRI }}^{k}-L f_{\text {ULRI }}^{k}}{U f_{\text {ULRI }}^{k}-L f_{\text {ULRI }}^{k}} & \text { if } \quad L f_{\text {ULRI }}^{k}<f_{\text {ULRI }}^{k}<U f_{\text {ULRI }}^{k} \\
0 & \text { if } & f_{\text {ULRI }}^{k} \geq U f_{\text {ULRI }}^{k}
\end{array}\right.
$$

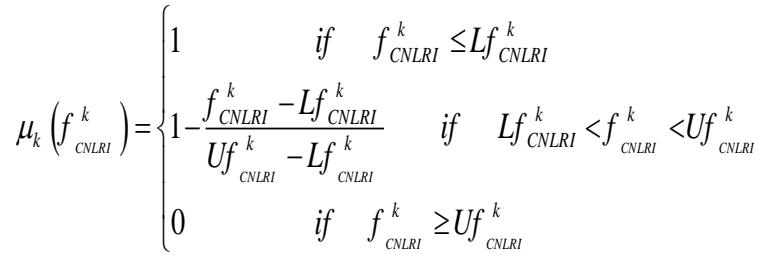

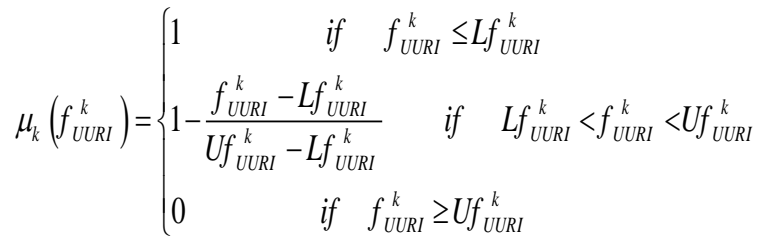

$$
\mu_{k}\left(f_{\text {CNURI }}^{k}\right)=\left\{\begin{array}{l}
1 \quad \text { if } f_{\text {CNURI }}^{k} \leq L f_{\text {CNURI }}^{k} \\
1-\frac{f_{\text {CNURI }}^{k}-L f_{\text {CNURI }}^{k}}{U f_{\text {CNURI }}^{k}-L f_{\text {CNURI }}^{k}} \text { if } \quad L f_{\text {CNURI }}^{k}<f_{\text {CNURI }}^{k}<U f_{\text {CNURI I }}^{k} \\
0 \quad \text { if } f_{\text {CNURI }}^{k} \geq U f_{\text {CNURI }}^{k}
\end{array}\right.
$$

$\operatorname{Max} \mu$

subject to

$$
\begin{aligned}
& f_{U L L I}^{k}+\mu\left(U f_{U R L I}^{k}-L f_{U R R I}^{k}\right) \leq U f_{U L R l}^{k}
\end{aligned}
$$

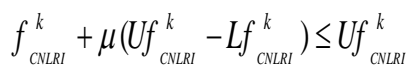

$$
\begin{aligned}
& f_{U U R I}^{k}+\mu\left(U f_{U U R I}^{k}-L f_{U U R I}^{k}\right) \leq U f_{U U R I}^{k} \\
& f_{\text {cower }}^{k}+\mu\left(U f_{\text {cuvel }}^{k}-L f_{\text {cverl }}^{k}\right) \leq U f_{\text {cyver }}^{k} \\
& \sum_{j=1}^{n} x_{i j} \leq a_{i}^{d}, \sum_{j=1}^{n} x_{i j} \geq a_{i}^{c}, i=1,2, \ldots, m \\
& \sum_{j=1}^{n} x_{i j} \leq b_{j}^{d}, \sum_{j=1}^{n} x_{i j} \geq b_{j}^{c}, j=1,2, \ldots, n \\
& \sum_{i=1}^{m} a_{i}^{c}=\sum_{j=1}^{n} b_{j}^{c}, i=1,2, \ldots, m, j=1,2, \ldots, n \\
& \sum_{i=1}^{m} a_{i}^{d}=\sum_{j=1}^{n} b_{j}^{d}, i=1,2, \ldots, m, j=1,2, \ldots, n \\
& x_{i j} \geq 0 \forall i, j, \mu \geq 0
\end{aligned}
$$

\section{ILLUSTRATIVE EXAMPLES}

To solve the 3 examples(roughness in objective function only ,roughness in feasible region only and fully roughness) beasd on the the right limit, the center and the half-width of each rough interval using possibly region. And all solutions are deterministic.

In order to illustrate the solution of the first one which cost of problem given as rough and the DM is quite sure of the quantities exist in demands and capacities of supplies. i.e. the roughness exist only in the objective function while the constraints are deterministic. 


\section{Example 1}

$\min f^{1}=\sum_{i=1}^{m} \sum_{j=1}^{n} c_{i j}^{R I 1} x_{i j}$

$\min f^{2}=\sum_{i=1}^{m} \sum_{j=1}^{n} c_{i j}^{R I 2} x_{i j}$

subject to

$$
\begin{gathered}
\sum_{j=1}^{4} x_{1 j}=8, \sum_{j=1}^{4} x_{2 j}=19, \sum_{j=1}^{4} x_{3 j}=17 \\
\sum_{i=1}^{3} x_{i 1}=11, \sum_{i=1}^{3} x_{i 2}=3, \sum_{i=1}^{3} x_{i 3}=14, \sum_{i=1}^{3} x_{i 4}=16 \\
x_{i j} \geq 0
\end{gathered}
$$

Where the $C_{i j}^{R I 1}$ and $C_{i j}^{R I 2}$

$C_{i j}^{R 1}=\left(\begin{array}{cccc}{[[6,7]:[5,9]]} & {[[6,7]:[5,9]]} & {[[5,7]:[4,8]]} & {[[6,7]:[5,9]]} \\ {[[9,11]:[8,12]]} & {[[15,17]:[14,18]]} & {[[16,17]:[14,19]]} & {[[18,21]:[17,22]]} \\ {[[11,13]:[10,14]]} & {[[3,5]:[2,6]]} & {[[9,11]:[8,12]]} & {[[3,4]:[1,5]]}\end{array}\right)$ $C_{i j}^{R 2}=\left(\begin{array}{cccc}{[[7,9]:[6,10]]} & {[[10,11]:[8,12]]} & {[[11,12]:[10,13]]} & {[[4,6]:[3,6]]} \\ {[[6,8]:[5,9]]} & {[[9,10]:[7,11]]} & {[[5,7]:[4,8]]} & {[[7,9]:[6,11]]} \\ {[[8,10]:[7,11]]} & {[[13,15]:[12,16]]} & {[[8,10]:[7,11]]} & {[[0,11]:[8,12]]}\end{array}\right)$

In order to solve such example we construct the model as in (8) based on the bounded of the rough interval and canter as follows:

$$
\begin{aligned}
\text { Min } \quad f_{\text {ULRI }}^{1}(x) & =7 x_{11}+7 x_{12}+7 x_{13}+7 x_{14}+11 x_{21}+17 x_{22} \\
& +17 x_{23}+21 x_{24}+13 x_{31}+5 x_{32}+11 x_{33}+4 x_{34} \\
\text { Min } \quad f_{\text {CNLRI }}^{1}(x) & =6.5 x_{11}+6.5 x_{12}+6 x_{13}+6.5 x_{14}+10 x_{21}+16 x_{22} \\
& +16.5 x_{23}+19.5 x_{24}+12 x_{31}+4 x_{32}+10 x_{33}+3.5 x_{34} \\
\text { Min } \quad f_{\text {UURI }}^{1}(x) & =9 x_{11}+9 x_{12}+8 x_{13}+9 x_{14}+10 x_{21}+18 x_{22}+19 x_{23} \\
& +22 x_{24}+14 x_{31}+6 x_{32}+12 x_{33}+5 x_{34} \\
\text { Min } \quad f_{\text {CNURI }}^{1}(x) & =7 x_{11}+7 x_{12}+6 x_{13}+7 x_{14}+10 x_{21}+16 x_{22} \\
& +16.5 x_{23}+19.5 x_{24}+12 x_{31}+4 x_{32}+10 x_{33}+3 x_{34}
\end{aligned}
$$

Table 1: Payoff Table of Example 1

\begin{tabular}{lllllllll}
\hline & $f_{\text {ULRI }}^{1}$ & $f_{\text {CNLRI }}^{1}$ & $f_{\text {UURI }}^{1}$ & $f_{\text {CNURI }}^{1}$ & $f_{\text {ULRI }}^{2}$ & $f_{\text {CNLRI }}^{2}$ & $f_{\text {UURI }}^{2}$ & $f_{\text {CNURI }}^{2}$ \\
\hline$X^{\text {ULRII }}$ & 380 & 351 & 436 & 344 & 429 & 397 & 469 & 382 \\
$X^{\text {CNLRII }}$ & 382 & 349 & 410 & 341 & 437 & 406 & 473 & 397 \\
$X^{\text {UURII }}$ & 382 & 349 & 410 & 341 & 437 & 406 & 473 & 397 \\
$X^{\text {CNURII }}$ & 382 & 349 & 410 & 341 & 437 & 406 & 473 & 397 \\
$X^{\text {ULRI2 }}$ & 516 & 487 & 582 & 487 & 370 & 322.5 & 416 & 322
\end{tabular}

Min $f_{\text {ULRI }}^{2}(x)=9 x_{11}+11 x_{12}+12 x_{13}+6 x_{14}+8 x_{21}+10 x_{22}$ $+7 x_{23}+9 x_{24}+10 x_{31}+15 x_{32}+10 x_{33}+11 x_{34}$

Min $f_{\text {CNLRI }}^{2}(x)=8 x_{11}+10.5 x_{12}+11.5 x_{13}+5 x_{14}+7 x_{21}+9.5 x_{22}$ $+6 x_{23}+8 x_{24}+8 x_{31}+14 x_{32}+9 x_{33}+10.5 x_{34}$

Min $f_{\text {UURI }}^{2}(x)=10 x_{11}+12 x_{12}+13 x_{13}+6 x_{14}+9 x_{21}+11 x_{22}$ $+8 x_{23}+11 x_{24}+11 x_{31}+16 x_{32}+11 x_{33}+12 x_{34}$

Min $f_{\text {CNURI }}^{2}(x)=8 x_{11}+10 x_{12}+11.5 x_{13}+4.5 x_{14}+7 x_{21}+9 x_{22}$ $+6 x_{23}+8.5 x_{24}+9 x_{31}+14 x_{32}+9 x_{33}+10 x_{34}$

subject to

$$
\begin{aligned}
& x_{11}+x_{12}+x_{13}+x_{14}=8 \\
& x_{21}+x_{22}+x_{23}+x_{24}=19 \\
& x_{31}+x_{32}+x_{33}+x_{34}=17 \\
& x_{11}+x_{21}+x_{31}=11 \\
& x_{12}+x_{22}+x_{32}=3 \\
& x_{13}+x_{23}+x_{33}=14 \\
& x_{14}+x_{24}+x_{34}=16 \\
& x_{i j} \geq 0 \quad \forall i, \forall j,
\end{aligned}
$$

In order to solve the above model we have to solve each objective function separately and calculate the remaining objectives at the resulted as presented on Table 1 .

As the 1, 2, 3 steps, the solution each single objective transportation problem where

$$
\begin{aligned}
& \quad X=\left(x_{11}, x_{12}, x_{13}, x_{14}, x_{21}, x_{22}, x_{23}, x_{24}, x_{31}, x_{32}, x_{33}, x_{34}\right) \\
& X^{\text {ULRI } 1}=(0,2,6,0,11,0,8,0,0,1,0,16) \\
& X^{\text {CNLRI1 }}=(0,0,8,0,11,2,6,0,0,1,0,16) \\
& X^{\text {UURI1 }}=(0,0,8,0,11,2,6,0,0,1,0,16) \\
& X^{\text {CNURI1 }}=(0,0,8,0,11,2,6,0,0,1,0,16) \\
& X^{\text {ULRI2 }}=(0,0,0,8,2,3,14,0,9,0,0,8) \\
& X^{\text {CNLRI } 2}=(0,0,0,8,0,3,14,2,11,0,0,6) \\
& X^{\text {UURI2 }}=(0,0,0,8,2,3,14,0,9,0,0,8) \\
& X^{\text {CNURI } 2}=(0,0,0,8,2,3,14,0,9,0,0,8)
\end{aligned}
$$

Step 4: Find the best lower and the worst upper for each objective (payoff Table) see table 1 


\begin{tabular}{lllllllll}
$X^{\text {CNLRI2 }}$ & 554 & 523 & 620 & 524 & 370 & 319.5 & 408 & 323 \\
$X^{\text {UURI2 }}$ & 516 & 487 & 572 & 487 & 370 & 322.5 & 406 & 322 \\
$X^{\text {CNURI2 }}$ & 516 & 487 & 582 & 487 & 370 & 319.5 & 406 & 322 \\
\hline Upper bound & 554 & 523 & 620 & 524 & 437 & 406 & 473 & 397 \\
Lower bound & 382 & 349 & 410 & 341 & 370 & 319.5 & 406 & 322 \\
The difference & 172 & 178 & 210 & 183 & 67 & 86.5 & 67 & 75
\end{tabular}

The linear model can be constructed based on the upper and lower bounds exist above as follows

$\max \omega$

\section{subject to}

$$
\begin{aligned}
& 7 x_{11}+7 x_{12}+7 x_{13}+7 x_{14}+11 x_{21}+17 x_{22}+17 x_{23} \\
+ & 21 x_{24}+13 x_{31}+5 x_{32}+11 x_{33}+4 x_{34}+172 \omega \leq 554 \\
& 6.5 x_{11}+6.5 x_{12}+6 x_{13}+6.5 x_{14}+10 x_{21}+16 x_{22}+16.5 x_{23} \\
+ & 19.5 x_{24}+12 x_{31}+4 x_{32}+10 x_{33}+3.5 x_{34}+174 \omega \leq 523 \\
9 & x_{11}+9 x_{12}+8 x_{13}+9 x_{14}+10 x_{21}+18 x_{22}+19 x_{23} \\
+ & 22 x_{24}+14 x_{31}+6 x_{32}+12 x_{33}+5 x_{34}+210 \omega \leq 620 \\
& 7 x_{11}+7 x_{12}+6 x_{13}+7 x_{14}+10 x_{21}+16 x_{22}+16.5 x_{23} \\
+ & 19.5 x_{24}+12 x_{31}+4 x_{32}+10 x_{33}+3 x_{34}+183 \omega \leq 524 \\
& 9 x_{11}+11 x_{12}+12 x_{13}+6 x_{14}+8 x_{21}+10 x_{22}+7 x_{23} \\
+ & 9 x_{24}+10 x_{31}+15 x_{32}+10 x_{33}+11 x_{34}+67 \omega \leq 473 \\
8 & x_{11}+10.5 x_{12}+11.5 x_{13}+5 x_{14}+7 x_{21}+9.5 x_{22}+6 x_{23} \\
+ & 8 x_{24}+8 x_{31}+14 x_{32}+9 x_{33}+10.5 x_{34}+86.5 \omega \leq 406 \\
10 & x_{11}+12 x_{12}+13 x_{13}+6 x_{14}+9 x_{21}+11 x_{22}+8 x_{23} \\
+ & 11 x_{24}+11 x_{31}+16 x_{32}+11 x_{33}+12 x_{34}+67 \omega \leq 473 \\
8 & x_{11}+10 x_{12}+11.5 x_{13}+4.5 x_{14}+7 x_{21}+9 x_{22}+6 x_{23} \\
+ & 8.5 x_{24}+9 x_{31}+14 x_{32}+9 x_{33}+10 x_{34}+75 \omega \leq 397 \\
& x_{11}+x_{12}+x_{13}+x_{14}=8 \\
& x_{21}+x_{22}+x_{23}+x_{24}=19 \\
& x_{31}+x_{32}+x_{33}+x_{34}=17 \\
& x_{11}+x_{21}+x_{31}=11 \\
& x_{12}+x_{22}+x_{32}=3 \\
& x_{13}+x_{23}+x_{33}=14 \\
& x_{14}+x_{24}+x_{34}=16 \\
& x_{i j} \quad \forall i, \forall j, \\
&
\end{aligned}
$$

By solving the above model the Pareto optimal solution of the problem is obtained as follows

$X=(0,0,0,8,11,1,7,0,0,2,7,8)$

$F^{l}=[[367,432]:[308.491]], F^{2}=[[304,383]:[251.419]]$

\section{Example 2}

Minimize $f^{1}=\sum_{i=1}^{3} \sum_{j=1}^{4} c_{i j}^{1} x_{i j} \quad, f^{2}=\sum_{i=1}^{3} \sum_{j=1}^{4} c_{i j}^{2} x_{i j}$

where $c^{1}$ and $c^{2}$ are

$$
C^{1}=\left[\begin{array}{cccc}
7 & 7 & 6 & 7 \\
10 & 16 & 17 & 20 \\
12 & 4 & 10 & 3
\end{array}\right], C^{2}=\left[\begin{array}{cccc}
8 & 10 & 11 & 5 \\
7 & 9 & 6 & 8 \\
9 & 14 & 9 & 10
\end{array}\right]
$$

subject to

$$
\begin{aligned}
\sum_{j=1}^{4} x_{1 j} & =[[7,9]:[6,10]], \sum_{j=1}^{4} x_{2 j}=[[17,21]:[16,22]] \\
\sum_{j=1}^{4} x_{3 j} & =[[16,18]:[15,19]] \\
\sum_{i=1}^{3} x_{i 1} & =[[10,12]:[9,13]], \sum_{i=1}^{3} x_{i 2}=[[2,4]:[1,5]] \\
\sum_{i=1}^{3} x_{i 3} & =[[13,15]:[12,16]], \sum_{i=1}^{3} x_{i 4}=[[15,17]:[15,17]] \\
x_{i j} & \geq 0
\end{aligned}
$$

Convert the rough constraints into deterministic constraints $\operatorname{Min} f^{1}(x)=7 x_{11}+7 x_{12}+6 x_{13}+7 x_{14}+10 x_{21}+16 x_{22}$ $+17 x_{23}+20 x_{24}+12 x_{31}+4 x_{32}+10 x_{33}+3 x_{34}$ $\operatorname{Min}^{2}(x)=8 x_{11}+10 x_{12}+11 x_{13}+5 x_{14}+7 x_{21}+9 x_{22}$ $+6 x_{23}+8 x_{24}+9 x_{31}+14 x_{32}+9 x_{33}+10 x_{34}$

subject to

$$
\begin{aligned}
& 6 \leq x_{11}+x_{12}+x_{13}+x_{14} \leq 10 \\
& 16 \leq x_{21}+x_{22}+x_{23}+x_{24} \leq 22 \\
& 15 \leq x_{31}+x_{32}+x_{33}+x_{34} \leq 19 \\
& 9 \leq x_{11}+x_{21}+x_{31} \leq 13 \\
& 1 \leq x_{12}+x_{22}+x_{32} \leq 5 \\
& 12 \leq x_{13}+x_{23}+x_{33} \leq 16 \\
& 15 \leq x_{14}+x_{24}+x_{34} \leq 17 \\
& \quad x_{i j} \geq 0 \quad \forall i, \forall j,
\end{aligned}
$$

As the 1, 2, 3 steps, the solution of each single objective transportation problem 
$X^{1}=(0,0,8,0,12,0,5,0,0,2,0,15)$

$X^{2}=(0,0,0,7,0,2,13,2,10,0,0,6)$

Find the best lower and the worst upper for each objective.

Table 2: Payoff Table example 2

\begin{tabular}{lll}
\hline & $f^{1}$ & $f^{2}$ \\
\hline$X^{I}$ & 306 & 380 \\
$X^{2}$ & 487 & 297 \\
\hline Upper & 487 & 380 \\
Lower & 306 & 297 \\
Difference & 181 & 83 \\
\hline
\end{tabular}

$\max \mu$

subject to

$$
\begin{gathered}
7 x_{11}+7 x_{12}+6 x_{13}+7 x_{14}+10 x_{21}+16 x_{22}+17 x_{23} \\
+20 x_{24}+12 x_{31}+4 x_{32}+10 x_{33}+3 x_{34}+181 \mu \leq 487 \\
8 x_{11}+10 x_{12}+11 x_{13}+5 x_{14}+7 x_{21}+9 x_{22}+6 x_{23} \\
+8 x_{24}+9 x_{31}+14 x_{32}+9 x_{33}+10 x_{34} 83 \mu \leq 380 \\
6 \leq x_{11}+x_{12}+x_{13}+x_{14} \leq 10 \\
16 \leq x_{21}+x_{22}+x_{23}+x_{24} \leq 22 \\
15 \leq x_{31}+x_{32}+x_{33}+x_{34} \leq 19 \\
9 \leq x_{11}+x_{21}+x_{31} \leq 13 \\
1 \leq x_{12}+x_{22}+x_{32} \leq 5 \\
12 \leq x_{13}+x_{23}+x_{33} \leq 16 \\
15 \leq x_{14}+x_{24}+x_{34} \leq 17 \\
x_{i j} \geq 0 \quad \forall i, j, \mu \geq 0
\end{gathered}
$$

the Pareto optimal solution of the problem using fuzzy technique is obtained as:

$X=(0,1,1,5,10,0,7,0,0,1,5,10) \quad F^{l}=356, \quad F^{2}=317$

\section{Example 3:}

$\operatorname{Min} f^{1}=\sum_{i=1}^{m} \sum_{j=1}^{n} c_{i j}^{R I 1} x_{i j}$

$\operatorname{Min} f^{2}=\sum_{i=1}^{m} \sum_{j=1}^{n} c_{i j}^{R I 2} x_{i j}$

subject to

$$
\begin{aligned}
\sum_{j=1}^{4} x_{1 j} & =[[7,9]:[6,10]], \sum_{j=1}^{4} x_{2 j}=[[17,21]:[16,22]] \\
\sum_{j=1}^{4} x_{3 j} & =[[16,18]:[15,19]], \\
\sum_{i=1}^{3} x_{i 1} & =[[10,12]:[9,13]], \sum_{i=1}^{3} x_{i 2}=[[2,4]:[1,5]] \\
\sum_{i=1}^{3} x_{i 3} & =[[13,15]:[12,16]], \sum_{i=1}^{3} x_{i 4}=[[15,17]:[15,17]] \\
x_{i j} & \geq 0
\end{aligned}
$$

where $c^{R I 1}$ and $c^{R I 2}$ are

$$
C_{i j}^{R 1}=\left(\begin{array}{cccc}
{[[6,7]:[5,9]]} & {[[6,7]:[5,9]]} & {[[5,7]:[4,8]]} & {[[6,7]:[5,9]]} \\
{[[9,11]:[8,12]]} & {[[15,17]:[14,18]]} & {[[16,17]:[14,19]]} & {[[18,21]:[17,22]]} \\
{[[11,13]:[10,14]]} & {[[3,5]:[2,6]]} & {[[9,11]:[8,12]]} & {[[3,4]:[1,5]]}
\end{array}\right)
$$

$C_{i j}^{R 2}=\left(\begin{array}{cccc}{[[7,9]:[6,10]]} & {[[10,11]:[8,12]]} & {[[11,12]:[10,13]]} & {[[4,6]:[3,6]]} \\ {[[6,8]:[5,9]]} & {[[9,10]:[7,11]]} & {[[5,7]:[4,8]]} & {[[7,9]:[6,11]]} \\ {[[8,10]:[7,11]]} & {[[13,15]:[12,16]]} & {[[8,10]:[7,11]]} & {[[0,11]:[8,12]]}\end{array}\right)$

Convert the rough objective into deterministic objective Min $f_{\text {ULRI }}^{1}(x)=7 x_{11}+7 x_{12}+7 x_{13}+7 x_{14}+11 x_{21}+17 x_{22}$ $+17 x_{23}+21 x_{24}+13 x_{31}+5 x_{32}+11 x_{33}+4 x_{34}$

Min $f_{\text {CNLRI }}^{1}(x)=6.5 x_{11}+6.5 x_{12}+6 x_{13}+6.5 x_{14}+10 x_{21}+16 x_{22}$ $+16.5 x_{23}+19.5 x_{24}+12 x_{31}+4 x_{32}+10 x_{33}+3.5 x_{34}$

Min $f_{\text {UURI }}^{1}(x)=9 x_{11}+9 x_{12}+8 x_{13}+9 x_{14}+10 x_{21}+18 x_{22}$ $+19 x_{23}+22 x_{24}+14 x_{31}+6 x_{32}+12 x_{33}+5 x_{34}$

Min $f_{\text {CNURI }}^{1}(x)=7 x_{11}+7 x_{12}+6 x_{13}+7 x_{14}+10 x_{21}+16 x_{22}$ $+16.5 x_{23}+19.5 x_{24}+12 x_{31}+4 x_{32}+10 x_{33}+3 x_{34}$

$\operatorname{Min} f_{\text {ULRI }}^{2}(x)=9 x_{11}+11 x_{12}+12 x_{13}+6 x_{14}+8 x_{21}+10 x_{22}$ $+7 x_{23}+9 x_{24}+10 x_{31}+15 x_{32}+10 x_{33}+11 x_{34}$

Min $f_{\text {CNLRI }}^{2}(x)=8 x_{11}+10.5 x_{12}+11.5 x_{13}+5 x_{14}+7 x_{21}+9.5 x_{22}$ $+6 x_{23}+8 x_{24}+8 x_{31}+14 x_{32}+9 x_{33}+10.5 x_{34}$

Min $f_{\text {UURI }}^{2}(x)=10 x_{11}+12 x_{12}+13 x_{13}+6 x_{14}+9 x_{21}+11 x_{22}$ $+8 x_{23}+11 x_{24}+11 x_{31}+16 x_{32}+11 x_{33}+12 x_{34}$

Min $f_{\text {CNURI }}^{2}(x)=8 x_{11}+10 x_{12}+11.5 x_{13}+4.5 x_{14}+7 x_{21}+9 x_{22}$ $+6 x_{23}+8.5 x_{24}+9 x_{31}+14 x_{32}+9 x_{33}+10 x_{34}$

Convert the rough constraints into deterministic constraints subject to

$$
\begin{aligned}
& 6 \leq x_{11}+x_{12}+x_{13}+x_{14} \leq 10 \\
& 16 \leq x_{21}+x_{22}+x_{23}+x_{24} \leq 22 \\
& 15 \leq x_{31}+x_{32}+x_{33}+x_{34} \leq 19 \\
& 9 \leq x_{11}+x_{21}+x_{31} \leq 13 \\
& 1 \leq x_{12}+x_{22}+x_{32} \leq 5 \\
& 12 \leq x_{13}+x_{23}+x_{33} \leq 16 \\
& 15 \leq x_{14}+x_{24}+x_{34} \leq 17 \\
& x_{i j} \geq 0 \quad \forall i, j
\end{aligned}
$$

As the 1, 2, 3 steps, the solution of each single objective transportation problem

$$
\begin{aligned}
& X^{\text {ULRI } 1}=(0,0,7,0,11,0,6,0,0,2,0,15) \\
& X^{\text {CNLRI 1 }}=(0,0,8,0,12,0,5,0,0,2,0,15) \\
& X^{\text {UURI 1 }}=(0,0,9,0,12,1,4,0,0,1,0,15) \\
& X^{\text {CNURI 1 }}=(0,0,8,0,12,0,5,0,0,2,0,15) \\
& X^{\text {ULRL2 }}=(0,0,0,7,0,2,13,2,10,0,0,6) \\
& X^{\text {CNLRI 2 }}=(0,0,0,7,0,2,13,2,10,0,0,6) \\
& X^{\text {UURI 2 }}=(0,0,0,7,2,2,13,0,8,0,0,8) \\
& X^{\text {CNURI 2 }}=(0,0,0,7,2,2,13,0,8,0,0,8)
\end{aligned}
$$

Find the best lower and the worst upper for each objective by solving the classical multiobjective transportation problem. 
Table 3: Payoff Table Example 3

\begin{tabular}{lllllllll}
\hline & $f_{\text {ULRI }}^{1}$ & $f_{\text {CNLRI }}^{1}$ & $f_{\text {UURI }}^{1}$ & $f_{\text {CNURI }}^{1}$ & $f_{\text {ULRI }}^{2}$ & $f_{\text {CNLRI }}^{2}$ & $f_{\text {UURI }}^{2}$ & $f_{\text {CNURI }}^{2}$ \\
\hline$X^{\text {ULRII }}$ & 342 & 311.5 & 367 & 304 & 416 & 382.5 & 443 \\
$X^{\text {CNLRII }}$ & 343 & 311 & 366 & 303.5 & 430 & 395.5 & 456 & 368 \\
$X^{\text {UURII }}$ & 345 & 312.5 & 365 & 305 & 431 & 397 & 455 & 380 \\
$X^{\text {CNURII }}$ & 382 & 311 & 366 & 303.5 & 430 & 395.5 & 456 & 380 \\
$X^{\text {ULRI2 }}$ & 500 & 472 & 556 & 472.5 & 337 & 291 & 472 & 294.5 \\
$X^{\text {CNLRI2 }}$ & 500 & 472 & 556 & 472.5 & 337 & 291 & 472 & 294.5 \\
$X^{\text {UURI2 }}$ & 462 & 436 & 514 & 435.5 & 337 & 294 & 370 & 293.5 \\
$X^{\text {CNURI2 }}$ & 462 & 436 & 514 & 435.5 & 337 & 294 & 370 & 293.5 \\
\hline Upper bound & 500 & 372 & 556 & 472.5 & 431 & 397 & 456 & 380 \\
Lower bound & 342 & 311 & 365 & 303.5 & 337 & 291 & 370 \\
The & 158 & 161 & 191 & 169 & 94 & 106 & 86 \\
difference & & & & & & & 293.5 \\
\hline
\end{tabular}

The linear model can be constructed Based on the upper and lower bounds exist above as follows: $\max \omega$

\section{subject to}

$$
\begin{aligned}
& 7 x_{11}+7 x_{12}+7 x_{13}+7 x_{14}+11 x_{21}+17 x_{22}+17 x_{23} \\
& +21 x_{24}+13 x_{31}+5 x_{32}+11 x_{33}+4 x_{34}+158 \omega \leq 500 \\
& 6.5 x_{11}+6.5 x_{12}+6 x_{13}+6.5 x_{14}+10 x_{21}+16 x_{22}+16.5 x_{23} \\
& +19.5 x_{24}+12 x_{31}+4 x_{32}+10 x_{33}+3.5 x_{34}+161 \omega \leq 372 \\
& 9 x_{11}+9 x_{12}+8 x_{13}+9 x_{14}+10 x_{21}+18 x_{22}+19 x_{23} \\
& +22 x_{24}+14 x_{31}+6 x_{32}+12 x_{33}+5 x_{34}+191 \omega \leq 556 \\
& 7 x_{11}+7 x_{12}+6 x_{13}+7 x_{14}+10 x_{21}+16 x_{22}+16.5 x_{23} \\
& +19.5 x_{24}+12 x_{31}+4 x_{32}+10 x_{33}+3 x_{34}+169 \omega \leq 472.5 \\
& 9 x_{11}+11 x_{12}+12 x_{13}+6 x_{14}+8 x_{21}+10 x_{22}+7 x_{23} \\
& +9 x_{24}+10 x_{31}+15 x_{32}+10 x_{33}+11 x_{34}+94 \omega \leq 431 \\
& 8 x_{11}+10.5 x_{12}+11.5 x_{13}+5 x_{14}+7 x_{21}+9.5 x_{22}+6 x_{23} \\
& +8 x_{24}+8 x_{31}+14 x_{32}+9 x_{33}+10.5 x_{34}+106 \omega \leq 397 \\
& 10 x_{11}+12 x_{12}+13 x_{13}+6 x_{14}+9 x_{21}+11 x_{22}+8 x_{23} \\
& +11 x_{24}+11 x_{31}+16 x_{32}+11 x_{33}+12 x_{34}+86 \omega \leq 456 \\
& 8 x_{11}+10 x_{12}+11.5 x_{13}+4.5 x_{14}+7 x_{21}+9 x_{22}+6 x_{23} \\
& +8.5 x_{24}+9 x_{31}+14 x_{32}+9 x_{33}+10 x_{34}+86.5 \omega \leq 380 \\
& 6 \leq x_{11}+x_{12}+x_{13}+x_{14} \leq 10 \\
& 16 \leq x_{21}+x_{22}+x_{23}+x_{24} \leq 22 \\
& 15 \leq x_{31}+x_{32}+x_{33}+x_{34} \leq 19 \\
& 9 \leq x_{11}+x_{21}+x_{31} \leq 13 \\
& 1 \leq x_{12}+x_{22}+x_{32} \leq 5 \\
& 12 \leq x_{13}+x_{23}+x_{33} \leq 16 \\
& 15 \leq x_{14}+x_{24}+x_{34} \leq 17 \\
& x_{i j} \geq 0, \forall i, j, \omega \geq 0 \\
& +800
\end{aligned}
$$

The Pareto optimal solution of the problem using fuzzy programming technique is obtained as following:

$X=(0,0,6,1,10,1,6,0,0,1,1,14)$

$F^{l}=\left([291,350]:[231.397), F^{2}=([310,389]:[275.428])\right.$

\section{CONCLUSION}

The different types of rough interval multiobjective transportation problem are introduced and the solution approach is presented. The concept of solving conventional interval programming combined with fuzzy programming is used to build the solution approach for RIMOTP. The proposed approach can be applied for solving different types of transportation problem such as rough interval fixed charge transportation problem, and rough interval solid transportation problem.

\section{REFERENCES}

[1] Abd El-Wahed W.F "A multi-objective transportation problem under fuzziness" Fuzzy Sets and Systems. 117 (2001) 27-33.

[2] A.K. Bit, M.P. Biswal, S.S. Alam, "Fuzzy programming approach to multicriteria decision making transportation problem", Fuzzy Sets and Systems. 50 (1992) 135-142.

[3] Ammar E. E. and Khalifa A. M " On Solving of Rough Interval multiobjective transportation problems" Journal of Advances in Physics. 7 (2014) 1233-1244.

[4] Das.S.K,Coswami.A and Alam.S. S" Multiobjective Transportation Problems with Interval Cost, Source and Destination Parameters" European Journal of operational research 117 (1999) 100-110.

[5] El-Sisy M. A.F, Osman MSA, and Eid MH, "On duality of Multiobjective convex Programming Problem ", Applied \&computational mathematics. 4(2015).

[6] Hongwei lu,Guohe H.and Li"An inexact rough -interval fuzzy liner programming method for generating conjunactive water-allocation strategies to agricultural irrigation system35(2011) 689-696.

[7] Lee S.M and Abd El-wahed.W.F, "Interactive fuzzy goal programming multiobjective transportation problem", Omega 34 (2006)158-166.

[8] Mohanty, S. P. and Subhakanta, D. "Transportation Programming under Uncertain Environment" International Journal of Engineering Research and Development 2013(7) 22-28.

[9] Osman. M.S, E.F. Lashein, Youness. E.A., and Attya T.E.M. "Rough Mathematical Programming Optimization," A Journal of Mathematical Programming and Operations Research, 58 (2009)1-8. 
[10] Osman .M.S.,El-Sherbiny. M.M. N,Khalifa.H.A,and. Hanan.H.H.Farag"Solving rough interval cost of transportation problem with source and destination parameters" The 49th annual Conference on statistics,Computer Sciences and Operations research (2014)17-29

[11] Osman .M.S., El-Sherbiny. M.M.N, Khalifa.H.A, and. Hanan.H.H.Farag" A Linear Programming Method for Solving Rough Interval Transportation Problem" The 50th annual Conference on statistics, Computer Sciences and Operations research (2015)1-15

[12] Pawlak.Z," Rough Sets", International Journal of Computer and Information Science 11 (1982) 341-356.
[13] Rebolledo M., Rough intervals - enhancing intervals for qualitative modeling of technical systems, Artif. Intell. 170 (2006) 667-685.

[14] Xu Jiuping, and Zhimiao T."A Class of Rough Multiple Objective Programming and its Application to Solid Transportation problem"; Information Sciences, 188 (2012) 215-235.

[15] Youness E.A."Characterizing Solution of Rough Programming Problems" Journal of Operational Research, 168 (2006)1019-1029.

[16] Zimmermann, H.J. "Fuzzy Programming and Linear Programming with Several Objective functions"; Fuzzy Sets and systems. 1 (1978) 45-55. 